\title{
Search for new loci and low-frequency variants influencing glioma risk by exome-array analysis
}

\author{
Ben Kinnersley ${ }^{1}$, Yoichiro Kamatani ${ }^{2}$, Marianne Labussière ${ }^{3}$, Yufei Wang ${ }^{1}$, Pilar Galan ${ }^{4}$, Karima Mokhtari ${ }^{3,5}$, \\ Jean-Yves Delattre ${ }^{3,5,6}$, Konstantinos Gousias ${ }^{7}$, Johannes Schramm ${ }^{7}$, Minouk J Schoemaker ${ }^{1}$, \\ Anthony Swerdlow ${ }^{8}$, Sarah J Fleming ${ }^{9}$, Stefan Herms ${ }^{10,11}$, Stefanie Heilmann ${ }^{10}$, Markus M Nöthen ${ }^{10}$, \\ Matthias Simon ${ }^{7}$, Marc Sanson ${ }^{3,5,6}$, Mark Lathrop ${ }^{2,5,12}$ and Richard S Houlston ${ }^{\star, 1}$
}

To identify protein-altering variants (PAVs) for glioma, we analysed Illumina HumanExome BeadChip exome-array data on 1882 glioma cases and 8079 controls from three independent European populations. In addition to single-variant tests we incorporated information on the predicted functional consequences of PAVs and analysed sets of genes with a higher likelihood of having a role in glioma on the basis of the profile of somatic mutations documented by large-scale sequencing initiatives. Globally there was a strong relationship between effect size and PAVs predicted to be damaging $\left(P=2.29 \times 10^{-49}\right)$; however, these variants which are most likely to impact on risk, are rare (MAF $<5 \%$ ). Although no single variant showed an association which was statistically significant at the genome-wide threshold a number represented promising associations - BRCA2: c.9976A $>$ T, p.(Lys3326Ter), which has been shown to influence breast and lung cancer risk (odds ratio $(O R)=2.3$, $P=4.00 \times 10^{-4}$ for glioblastoma (GBM)) and IDH2:c.782G $>$ A, p.(Arg261His) (OR = 3.21, $P=7.67 \times 10^{-3}$, for non-GBM). Additionally, gene burden tests revealed a statistically significant association for HARS2 and risk of GBM $\left(P=2.20 \times 10^{-6}\right)$. Genome scans of low-frequency PAVs represent a complementary strategy to identify disease-causing variants compared with scans based on tagSNPs. Strategies to lessen the multiple testing burden by restricting analysis to PAVs with higher priors affords an opportunity to maximise study power.

European Journal of Human Genetics (2016) 24, 717-724; doi:10.1038/ejhg.2015.170; published online 12 August 2015

\section{INTRODUCTION}

Gliomas account for $\sim 40 \%$ of all primary brain tumours and are diagnosed in around 26000 individuals in Europe each year. ${ }^{1,2}$ Gliomas are typically classified as being either glioblastoma (GBM) or non-GBM tumours (diffuse 'low-grade' glioma WHO grade I/II and anaplastic glioma WHO grade III tumours). ${ }^{3}$ Most gliomas carry a poor prognosis, with the most common type, GBM, typically having a median survival of 15 months. $^{2}$ The only environmental factor consistently shown to influence glioma risk is exposure to ionising radiation, ${ }^{2}$ which accounts for only a very small number of cases. Evidence for genetic predisposition to glioma is provided by rare inherited cancer syndromes including Turcot's and Li-Fraumeni syndromes, and neurofibromatosis. ${ }^{2,4}$ Collectively however they account for little of the 2-fold increased risk of glioma seen in relatives of patients. ${ }^{5}$

Much of the variation in genetic risk of glioma appears to be polygenic. Support for this proposal has come from genome-wide association studies (GWAS) which have identified common singlenucleotide polymorphisms (SNPs) at six loci influencing risk - 5p15.33 (TERT), 7p11.2 (EGFR, two regions), 8q24.21 (CCDC26), 9p21.3 (CDKN2A/CDKN2B), 11q23.3 (PHLDB1) and 20q13.33 (RTEL1). ${ }^{6-8}$
Despite the success of GWAS such studies are not optimally configured to identify low-frequency variants with stronger effects. Protein altering variants (PAVs), which alter the encoded amino acid sequence, are proportionally less prevalent than synonymous variants; however, such variants are a priori more likely to have a functional impact. Coupled with the observation that Mendelian disease susceptibility is generally caused by coding sequence changes ${ }^{9}$ suggests that association studies formulated around a gene-centric approach may be a powerful strategy for identifying disease-causing associations.

Although no rare recurrent PAV has thus far been shown to influence glioma risk the low-frequency variants NM_007194.3 (CHEK2):c.1100delC, p.(Thr367Metfs), NM_000059.3(BRCA2): c.9976A > T, p.(Lys3326Ter) and NM_000038.5(APC):c.3920T > A, p.(Ile1307Lys) confer 2- to 3-fold risks of breast, lung and colorectal cancers (CRC) respectively. ${ }^{10-12}$ Additionally the observation that the NM_001128425.1(MUTYH):c.536A > G, p.(Tyr179Cys) and NM_0011 28425.1(MUTYH):c.1187G >A, p.(Gly396Asp) variants cause recessive polyposis and $\mathrm{CRC}^{13}$ provides a precedent for rare recurrent variants having substantive effects on cancer risk.

The advent of next generation sequencing is allowing the cataloguing of recurrent coding variation, making the search for

\footnotetext{
${ }^{1}$ Division of Genetics and Epidemiology, The Institute of Cancer Research, Sutton, Surrey, UK; ${ }^{2}$ Foundation Jean Dausset-CEPH, Paris, France; ${ }^{3}$ Sorbonne Universités UPMC Univ Paris 06, INSERM CNRS, Paris, France; ${ }^{4}$ Université Paris 13 Sorbonne Paris Cité, Inserm (U557), Cnam, Bobigny, France; ${ }^{5}$ AP-HP, GH Pitié-Salpêtrière, Service de Neurologie Mazarin, Paris, France; ${ }^{6}$ Groupe Hospitalier Pitié-Salpêtrière, Paris, France; ${ }^{7}$ Department of Neurosurgery, University of Bonn Medical Center, Bonn, Germany; ${ }^{8}$ Division of Breast Cancer Research, The Institute of Cancer Research, Sutton, Surrey, UK; ${ }^{9}$ Centre for Epidemiology and Biostatistics, Faculty of Medicine and Health, University of Leeds, Leeds, UK; ${ }^{10}$ Institute of Human Genetics, University of Bonn, Bonn, Germany; ${ }^{11}$ Department of Biomedicine, Division of Medical Genetics, University of Basel, Basel, Switzerland; ${ }^{12}$ Department of Human Genetics, Génome Québec, McGill University, Montreal, QC, Canada

*Correspondence: Professor R Houlston, Division of Genetics and Epidemiology, The Institute of Cancer Research, Sutton, Surrey SM2 5NG, UK. Tel: +44 02087224175 ; E-mail: richard.houlston@icr.ac.uk

Received 7 January 2015; revised 31 May 2015; accepted 23 June 2015; published online 12 August 2015
} 
disease-causing PAVs on a genome-wide basis a viable proposition. Here we have investigated the contribution of recurrent coding variants to glioma by analysing 1882 cases and 8079 controls genotyped using the Illumina HumanExome BeadChip. To increase our power to identify disease-causing variants, we jointly tested groups of variants in a gene and incorporated information on the predicted functional consequences of PAVs. In addition we restricted our analysis to sets of genes with a higher likelihood of having a role in glioma on the basis of somatic mutation profile.

\section{MATERIALS AND METHODS}

\section{Subjects}

We analysed three non-overlapping case-control series of Northern European ancestry: the UK series comprised 605 glioma cases (63\% male; mean age at diagnosis 46 years) ascertained through the INTERPHONE Study ${ }^{14}$ with 5964 individuals from the 1958 Birth Cohort $\left(1958 \mathrm{BC} ;{ }^{15}\right)$ with no known personal history of cancer serving as a controls; the French series comprised 906 incident cases of glioma ascertained through the Service de Neurologie Mazarin, Groupe Hospitalier Pitié-Salpêtrière, Paris ${ }^{6}$ and 699 controls from the SU.VI.MAX (SUpplementation en VItamines et MinerauxAntioXydants) study of 12735 healthy subjects (women aged 35-60 years; men aged $45-60$ years); ${ }^{16}$ and the German series comprised 902 patients who underwent surgery for glioma at the University of Bonn Medical Centre, between 1996 and 2008, ${ }^{6}$ with 2400 healthy individuals from the Heinz-Nixdorf Recall study serving as controls. ${ }^{17}$ The study was conducted with ethical review board approval. Written informed consent was obtained from all subjects. DNA was extracted from EDTA-venous bloods using conventional methodologies and quantified using PicoGreen (Invitrogen Corp., Carlsbad, CA, USA).

\section{The exome array}

Briefly, the Illumina HumanExome-12v1_A Beadchip (Illumina, San Diego, CA, USA) includes 247870 markers focused on protein-altering variants identified from whole-exome sequencing DNA from $>12000$ individuals of multiple ethnicities and with multiple diseases/traits. In addition to 203310 PAVs, the array also features 4761 GWAS trait-associated SNPs, 2061 HLA tags, 3015 ancestry-informative markers, 4896 identity-by-descent estimation markers and 4139 random synonymous SNPs. Comprehensive details about the exome array are available at http://genome.sph.umich.edu/wiki/Exome_ Chip_Design.

\section{Exome array data availability}

Illumina HumanExome-12v1_A Beadchip array genotypes for individuals from the 1958BC are available from the European Genome-phenome Archive (EGA, http://www.ebi.ac.uk/ega/) under accession number EGAD00010000234. Similarly, array genotypes and phenotypes for the remaining datasets in this study have been deposited to EGA and are available under accession number EGAS00001001258.

\section{Genotyping and quality control}

Genotyping was conducted using Illumina HumanExome-12v1_A Beadchips in accordance with the manufacturer's recommendations (Illumina). Calling of genotypes was performed using Illumina GenomeStudio version 2011.1 software. Cluster boundaries were determined by calling study samples simultaneously. Probes were excluded if monomorphic in all datasets, had a call rate $<0.99$ in cases/controls in a series, the difference in uncalled genotypes between cases and controls was statistically significant $(P<0.05)$, if HardyWeinberg in controls $P<0.001$, or if non-autosomal (Supplementary Table 1). Samples were excluded if the call rate was $<0.99$, outlying heterozygosity ( $>3$ $\mathrm{SD}$ ), or if a discrepancy was observed between manifest sex and X-chromosome genotype. To assess the fidelity of genotyping we examined the concordance in 493 individuals from the $1958 \mathrm{BC},{ }^{15}$ which had also been sequenced ${ }^{18}$ using TruSeq capture in conjunction with Illumina HiSeq2000 technology, and a GATK2 ${ }^{\text {ref. }}{ }^{19}$ pipeline according to best practices. ${ }^{20,21}$ Genotypes were compared at genomic positions for which allele codings could be unambiguously assigned, excluding $257 \mathrm{~A} / \mathrm{T}$ and $\mathrm{C} / \mathrm{G}$ SNPs with $\mathrm{MAF}>0.40$.

\section{Statistical and bioinformatic analysis}

The main statistical and bioinformatics analyses were performed using PLINK v1.07(ref. 22) (Cambridge, MA, USA) and R v3.0 software (Vienna, Austria). Using the EIGENSOFT v4.2 smartpca package ${ }^{23,24}$ (Cambridge, MA, USA) we performed PCA to ensure comparability of case and controls. Individuals with non-Western European ancestry were identified and excluded by merging case and control data with 1000 Genomes project data. 100000 ld-pruned post-QC probes were used to compute eigenvectors in each cohort. Samples exhibiting significant deviations ( $6 \mathrm{SD}$ ) from the main case/control cluster up to the first 10 eigenvectors were classified as outliers and flagged for exclusion. Outlying population structure on the pruned data set was examined using fastSTRUCTURE ${ }^{25}$ if subsequent non-comparability was apparent between cases and controls. For first-degree relative pairs, the control from a casecontrol pair was removed; otherwise, the individual with the lower call rate was excluded. Associations were tested under an additive model. The adequacy of the case-control matching in each series and the possibility of differential genotyping of cases and controls was evaluated using quantile-quantile (Q-Q) plots of test statistics, restricting to variants with $\mathrm{MAF}>0.005$ to derive reasonable inflation estimates. Meta-analysis $P$-values and odds ratios (ORs) were calculated from per-study logistic regression beta values, under a fixedeffects model. We used Cochran's $Q$ statistic to test for heterogeneity; restricting the reporting of novel associations to those with $P_{\text {het }}>0.05$. We visually inspected genotype cluster plots for all reported variants. To explore variability in associations according to tumour histology, we derived ORs for all glioma, GBM and non-GBM. For the gene-based analysis, in addition to using the burden test which counts the number of minor alleles per gene per individual summed for all cases and controls, the sequence kernel association test (SKAT) was applied. ${ }^{26}$ Burden and SKAT gene-based tests were based on all post-QC non-monomorphic probes mapping to RefSeq genes imposing default weights and $\mathrm{MAF}<0.05$. Tests were implemented in plink-seq v0.09, and adjusted for study-specific effects by incorporating study as a covariate (using covar option). A single-variant association was declared significant if $P<1.40 \times 10^{-7}$ (Bonferroni correction for $118,815 \mathrm{PAVs}$, three tumour types). Gene-based association tests were considered significant if $P<2.49 \times 10^{-6}$ (10 045 genes, two tumour types). The power of our study to demonstrate an association for alleles with different MAFs was calculated assuming a multiplicative model. In all analyses a $P$-value of 0.05 was considered as representing statistical significance, after adjustment for multiple testing. Gene-set enrichment analysis (GSEA) of pre-ranked SKAT $P$-values, was performed on gene sets catalogued by the MSigDB v4.0 database (updated 31 May 2013) using GSEA software ${ }^{27}$ adopting default settings. Linkage disequilibrium (LD) $r^{2}$ metrics were estimated from UK10K whole-genome data. To restrict our analysis to genes with a higher likelihood of having a role in glioma on the basis of somatic mutation profile in tumours, we used MutSigCV version $1.4^{\text {ref. } 28}$ to identify genes harbouring more non-synonymous mutations than expected by chance given gene size, sequence context and mutation rate. Thresholding at false discovery rate $Q<0.1$ as advocated, ${ }^{28}$ MutSig scores were obtained for GBM and non-GBM tumours by interrogation of TCGA (The Cancer Genome Atlas) provisional data sets using cBioPortal. ${ }^{29}$ The Variant Effect Predictor (VEP; version 74$)^{30}$ was used to predict impact of variants on canonical Ensembl gene transcripts and functional consequences of missense variants according to SIFT, ${ }^{31}$ PolyPhen-2 ${ }^{\text {ref. }} 32$ and CONDEL. ${ }^{33}$ Computational modelling of the effect of amino acid changes on protein structure was carried out using the project HOPE server. ${ }^{34}$ To assess sequence conservation we used GERP ${ }^{35}$ and Phast_cons ${ }^{36}$ metrics.

\section{Quality control and array characteristics and performance}

We submitted 2413 cases and 3099 controls for genotyping. Twelve cases and eight controls failed genotyping (call rate $<0.95$ ). Five hundred and nineteen cases and 807 controls were excluded for the following reasons: outlying heterozygosity in rare (47 cases, 28 controls) and common (44 cases, 10 controls) SNPs; duplicates/close relatives (15 cases, 16 controls); sex discrepancies (29 cases, 10 controls); and non-European ancestry (49 cases, 5 controls; Supplementary Table 1 and Supplementary Figure 1A). Genotypes from 5964 individuals were available from the 1958BC (UK) series. We further excluded 169 individuals because of personal history of cancer (105), 


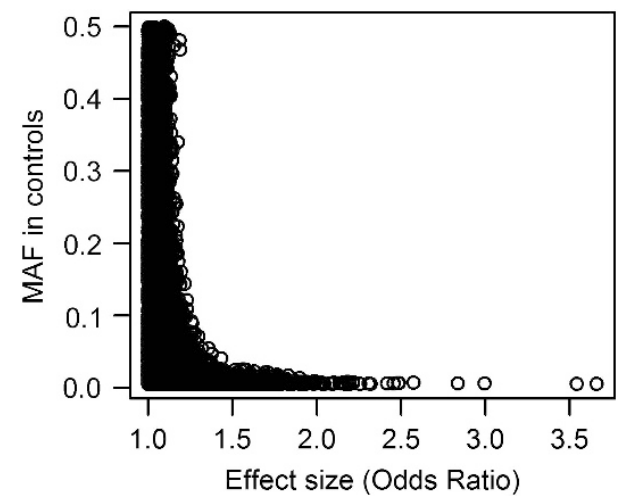

Figure 1 Relationship between effect size and minor allele frequency of PAVs.

outlying heterozygosity (16), sex discrepancy (22), duplicates/relatives (2) and non-European ancestry (24) (Supplementary Table 1). After excluding technical failures and imposing marker-level quality control, $90.2 \%$ of attempted markers were successfully genotyped (223 564/247 870). Concordance between genotype calls was assessed at 55955 sites in the 493 individuals for whom exome-chip and whole-exome sequence data were available (Supplementary Table 2). Overall the concordance was: $99.7 \%$, with $96.5 \%$, $96.0 \%$ and $99.8 \%$ when comparing minor homozygotes, heterozygotes and major homozygotes respectively. Restricting our analysis to 219771 autosomal probes, 84502 markers were monomorphic (38.5\%). Q-Q plots of association test statistics showed there was minimal inflation in the UK and French series $(\lambda=1.04$ and 1.05; Supplementary Figure 2A and B). In the German series, $\lambda$ was 1.17 (Supplementary Figure 2C). Using fastSTRUCTURE ${ }^{25}$ to impose two populations within the German series and retaining only individuals with $>80 \%$ membership of the larger population (2083 individuals, 488 cases and 797 controls; Supplementary Table 1 and Supplementary Figure 1B) $\lambda$ was 1.058 ensuring subsequent analysis was less biased by any ancestral discordance between cases and controls (Supplementary Figure 2D). Post-QC data on 1882 cases and 8079 controls were available for analysis.

\section{RESULTS}

Single-variant associations

In total 135269 variants (MAF>0.0) were taken forward for association testing in 1882 cases and 8079 controls. Genotypes for previously identified glioma GWAS risk SNPs or their proxies (ie, $r^{2}>0.8$ ) were available for $5 \mathrm{p} 15.33,7 \mathrm{p} 11.2,8 \mathrm{q} 24.21,9 \mathrm{p} 21.3$, $11 \mathrm{q} 23.3$ and $20 \mathrm{q} 13.33$ risk loci. ${ }^{6-8}$ OR and tumour subtype-specific associations were consistent with those previously documented (Supplementary Table 3).

To assess the impact of recurrent variants exerting a putative effect on protein function, we restricted our analysis to 118815 variants; 110625 missense, 5324 splice-site altering, 2616 stop gain, 168 uRNA targets and 82 indels. The MAF distribution was highly skewed towards very low-frequency variants (Supplementary Figure 3), with $80.4 \%$ ( $n=95488)$ of variants successfully genotyped having a control $\mathrm{MAF} \leq 0.005 ; \quad 4.0 \% \quad(n=4,764) \quad$ with $\mathrm{MAF}=0.05-0.01 ; \quad 6.4 \%$ $(n=7546)$ with $\mathrm{MAF}=0.01-0.05 ;$ and $9.3 \% \quad(n=11017)$ with MAF $>0.05$.

In the combined analysis of all PAVs the strongest association for risk of glioma was provided by rs593818 responsible for the XM_006722850.1(CYP4F12):c.1117A > G, p.(Ser373Gly) amino acid change $\left(P=1.24 \times 10^{-5}\right)$, albeit non-significant on a genome-wide basis (Supplementary Table 4). Similarly in the stratified analysis no single variant showed a globally significant association with either GBM or non-GBM tumours (Supplementary Table 4).

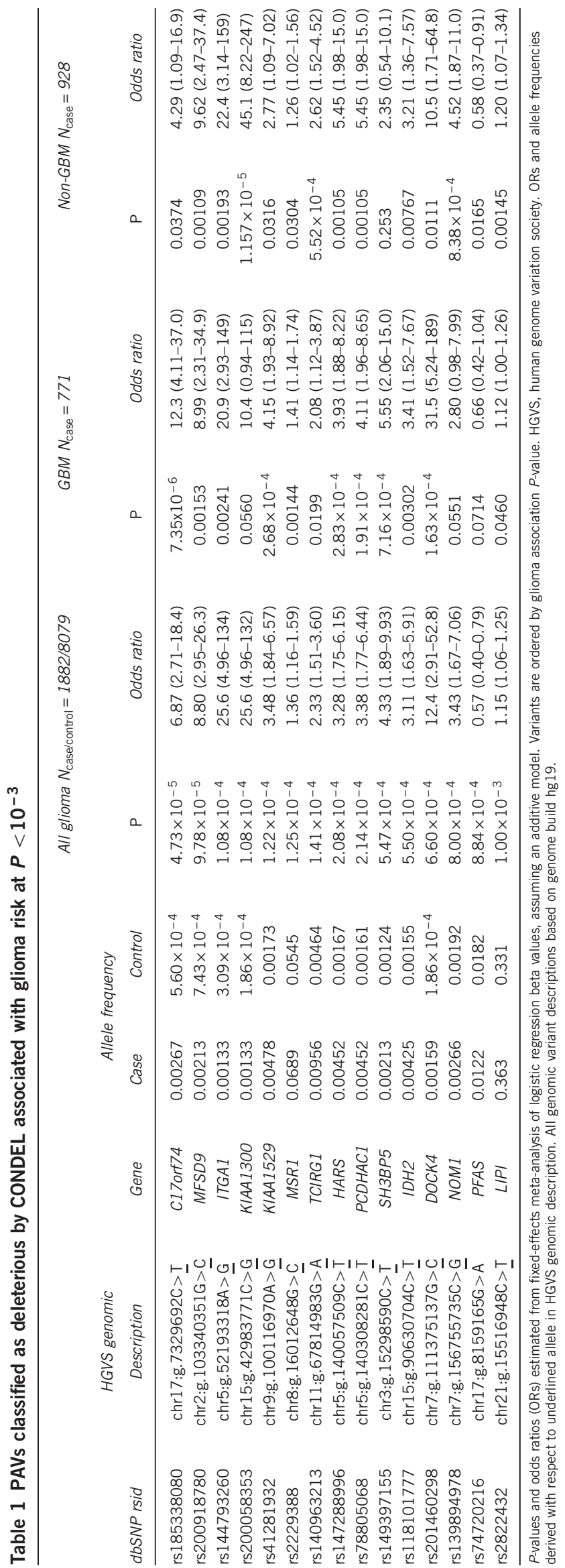

European Journal of Human Genetics 
Figure 1 shows the relationship between effect size (measured by OR, taking the reciprocal or ORs <1.0) and MAF for 118,815 PAVs, those SNPs characterized by low MAF tending to have a higher probability of conferring more substantive risks.

To restrict our analytical space, we analysed the data set incorporating information on the predicted functional consequences of these PAVs. Of the 104321 PAVs genotyped by the exome array for which CONDEL annotations could be obtained, the majority $(64.1 \%)$ are predicted to be neutral $(n=66841)$, and $35.9 \%$ deleterious $(n=37$ 480). Fifteen PAVs predicted to be deleterious showed an association with glioma risk at the $P<10^{-3}$ threshold (Table 1). To investigate whether PAVs predicted to be functionally deleterious were enriched for stronger effects on glioma risk, we compared the distribution of effect size (as measured by ORs) in the two CONDEL prediction categories (Table 2). There was strong evidence of a relationship

Table 2 Classification of PAVs with MAF $>0.005$ by Condel prediction, stratified by effect size in glioma

\section{Condel prediction}

\begin{tabular}{lcccc} 
Effect size $^{\mathrm{a}}$ & Neutral & Deleterious & Unknown & Total \\
\hline$<1.05$ & $6687(44.2 \%)$ & $1762(34.9 \%)$ & $1389(44.9 \%)$ & $9838(42.3 \%)$ \\
$1.05-1.10$ & $3603(23.8 \%)$ & $1125(22.3 \%)$ & $788(25.5 \%)$ & $5516(23.7 \%)$ \\
$1.10-1.20$ & $2648(17.5 \%)$ & $1086(21.5 \%)$ & $500(16.2 \%)$ & $4234(18.2 \%)$ \\
$1.20-1.50$ & $1868(12.4 \%)$ & $932(18.5 \%)$ & $352(11.4 \%)$ & $3152(13.6 \%)$ \\
$1.50-2.00$ & $287(1.9 \%)$ & $136(2.7 \%)$ & $59(1.9 \%)$ & $482(2.1 \%)$ \\
$2.00-3.00$ & $19(0.1 \%)$ & $8(0.2 \%)$ & $6(0.2 \%)$ & $33(0.1 \%)$ \\
$>3.00$ & $0(0.0 \%)$ & $1(0.02 \%)$ & $1(0.03 \%)$ & $2(0.0 \%)$ \\
Total & 15112 & 5050 & 3095 & 23257
\end{tabular}

a Measured by odds ratio (taking the reciprocal for $\mathrm{OR}<1.0$ ).

${ }^{\mathrm{b}} P_{\text {trend }}=2.29 \times 10^{-49}$ (Deleterious vs neutral; OR $\mathrm{R}_{\text {trend }}=1.22,95 \% \mathrm{Cl}: 1.19-1.26$ ). between increasing effect size and prediction of the PAV being deleterious. For PAVs with control MAF $>0.005$ predicted to be deleterious there was an OR increase of 1.22 compared with neutral PAVs (95\% confidence interval (CI): $1.19-1.26, P_{\text {trend }}=2.29 \times 10^{-49}$, Table 2). Overall, PAVs classified as damaging by CONDEL were 1.43fold more likely to be associated with effect sizes $\geq 1.5$ than PAVs classified as neutral $\left(P=4.59 \times 10^{-4}, \mathrm{OR}=1.43,95 \% \mathrm{CI}=1.17-1.74\right)$.

We further stratified our analysis to variants in genes that are significantly mutated in GBM and non-GBM glioma, as well as being nominally associated with glioma risk $(P<0.05)$. This identified 11 variants also significantly associated with GBM and five with non-GBM glioma (Table 3). Of interest is NM_002168.3(IDH2): c.782G $>$ A, p.(Arg261His) (rs118101777, non-GBM OR=3.21, $P=7.7 \times 10^{-3}$ ), which is predicted to be deleterious by CONDEL and is highly evolutionarily conserved (PhastCons $=1.00$, GERP $=5.84$ ).

A number of rare variants recognised to have pleiotropic effects on cancer risk are featured on the Illumina Exome Array (Table 4). For example, NM_000059.3(BRCA2):c.9976A > T, p.(Lys3326Ter) (rs11571833), which increases breast and lung cancer risk, ${ }^{10,37}$ NM_007194.3(CHEK2):c.470T > C, p.(Ile157Thr) (rs17879961), which increases breast cancer and CRC risk but decreases lung cancer risk, ${ }^{10,12,38}$ and NM_032043.2(BRIP1):c.139C > A, p.(Pro47Ala) (rs28903098), which has been implicated in familial breast and ovarian cancer. ${ }^{39}$ Given that such variants are a priori strong candidates for influencing the development of cancer, we examined the relationship between rs11571833, rs17879961 and rs28903098 and glioma (Table 5). For all glioma, BRCA2 p.(Lys3326Ter) carrier status conferred an OR of $1.76(P=0.0026)$, principally associated with GBM $\left(\mathrm{OR}=2.3, P=4.0 \times 10^{-4}\right)$. Although no association was shown for CHEK2 p.(Ile157Thr), BRIP1 p.(Pro47Ala) carrier status conferred an OR of $3.83(P=0.048)$ (Table 4$)$.

Table 3 Protein altering variants (PAVs) in genes significantly mutated in GBM and non-GBM Gliomas

\begin{tabular}{|c|c|c|c|c|c|c|c|c|}
\hline \multirow[b]{2}{*}{$d b S N P$ rsid } & \multirow[b]{2}{*}{ HGVS genomic description } & \multirow[b]{2}{*}{ Gene } & \multirow[b]{2}{*}{ Mutsig Q } & \multirow[b]{2}{*}{ Control allele frequency } & \multirow{2}{*}{\multicolumn{2}{|c|}{$\begin{array}{l}\text { GBM } \\
\text { Odds ratio }\end{array}$}} & \multicolumn{2}{|c|}{ Non-GBM } \\
\hline & & & & & & & $\mathrm{P}$ & Odds ratio \\
\hline \multicolumn{9}{|l|}{$G B M$} \\
\hline rs72658163 & chr7:g.94049588G > & COL1A2 & 0.0157 & 0.00204 & 0.0191 & $3.00(1.20-7.52)$ & & \\
\hline rs11569729 & chr4:g.70592915G>A & SULT1B1 & 0.00279 & 0.00159 & 0.0208 & 3.22 (1.19-8.68) & & \\
\hline rs121908919 & chr2:g.167138296T $>\underline{\mathrm{C}}$ & SCN9A & 0.0623 & 0.00233 & 0.0229 & $2.76(1.15-6.64)$ & & \\
\hline rs12364102 & chr11:g.56949691G $>\underline{A}$ & LRRC55 & 0.0223 & 0.126 & 0.0244 & $0.82(0.69-0.97)$ & & \\
\hline rs201984007 & chr2:g.167128917A $>\underline{G}$ & SCN9A & 0.0623 & $5.10 \times 10^{-4}$ & 0.0263 & $5.97(1.23-28.9)$ & & \\
\hline rs112884419 & chrl:g.158582637C $>\underline{A}$ & SPTA1 & $1.85 \times 10^{-9}$ & 0.00210 & 0.0318 & $2.64(1.09-6.38)$ & & \\
\hline rs144312303 & chr5:g.67586574G $>$ T & PIK3R1 & 0.000 & $2.84 \times 10^{-4}$ & 0.0320 & $20.8(1.30-334)$ & & \\
\hline rs149858889 & chr7:g.94050334C> & COL1A2 & 0.0157 & $1.70 \times 10^{-4}$ & 0.0336 & $13.6(1.23-150)$ & & \\
\hline rs140336416 & chr7:g.93116243A $>\bar{G}$ & $C A L C R$ & 0.0079 & $2.27 \times 10^{-4}$ & 0.0336 & $13.6(1.23-150)$ & & \\
\hline rs140857588 & chr5:g.19571925T $>\underline{\mathrm{C}}$ & $\mathrm{CDH} 18$ & $4.15 \times 10^{-5}$ & $2.27 \times 10^{-4}$ & 0.0401 & $5.93(1.08-32.5)$ & & \\
\hline rs71428908 & chr2:g.167160752G $>\underline{C}$ & SCN9A & 0.0623 & 0.00181 & 0.0440 & $2.70(1.03-7.10)$ & & \\
\hline \multicolumn{9}{|l|}{ Non-GBM } \\
\hline rs12442879 & chr15:g.57524982G $>\underline{A}$ & TCF 12 & $7.04 \times 10^{-4}$ & 0.0323 & & & $2.35 \times 10^{-4}$ & $1.65(1.26-2.14)$ \\
\hline rs118101777 & chr15:g.90630704C $>\bar{T}$ & $\mathrm{IDH} 2$ & $2.01 \times 10^{-12}$ & 0.00147 & & & 0.00767 & $3.21(1.36-7.57)$ \\
\hline rs72470545 & chr2:g.74759825G $>\underline{A}$ & HTRA2 & $1.01 \times 10^{-4}$ & 0.00335 & & & 0.0126 & $2.32(1.20-4.49)$ \\
\hline rs140596855 & chr15:g.90628584C $>\bar{T}$ & IDH2 & $2.01 \times 10^{-12}$ & $2.84 \times 10^{-4}$ & & & 0.0284 & $22.3(1.39-357)$ \\
\hline rs114905908 & chr4:g.162577630A $>\bar{T}$ & FSTL5 & 0.0078 & $3.40 \times 10^{-4}$ & & & 0.0493 & $11.1(1.01-123)$ \\
\hline
\end{tabular}

Shown are genes with meta-analysis $P$-values $<0.05$ and MutSig false discovery rate $Q$ values $<0.1$ for the relevant tumour type. HGVS, human genome variation society. Odds ratios and allele frequencies derived with respect to underlined allele in HGVS genomic description. All genomic variant descriptions based on genome build hg19. 
Gene and gene-set-based tests

As the majority of individual variants typed are very rare (median MAF $=3.7 \times 10^{-4}$ ), we assessed the burden of 70526 variants across 10045 genes. HARS2 showed an exome-wide significant association with GBM (Burden $P=2.00 \times 10^{-6}$, SKAT $P=1.03 \times 10^{-5}$, Table 5). Although not attaining exome-wide statistical significance, further gene-based tests revealed a number of genes that were both significantly mutated in glioma tumours as well as possessing a germline variant burden (Table 5).

To gain further insight into the nature of the biological pathways impacting on glioma susceptibility, we performed GSEA using SKAT association $P$-values (Supplementary Table 5 ). This revealed a number of gene sets that were positively or negatively enriched for genes associated with glioma (ie, $P_{G S E A}<0.05$ ). GBM glioma showed positive enrichment for genes involved in amino acid and nucleotide metabolism, and non-GBM glioma showed positive enrichment for genes involved in cell growth and development, however the majority of gene sets had an FDR $Q>0.25$.

\section{DISCUSSION}

GWAS have become a powerful tool to identify susceptibility variants for cancer. However since the tagSNPs used in GWAS are generally not themselves candidates for causality, identification of the functional variant at a locus generally poses a significant challenge. An alternative approach is to target sequence variation, which a priori, is more likely to impact on disease status. Alleles that are functionally deleterious will tend to be selected against and thus underrepresented at high frequencies, an assertion supported by the observation of a relationship between putative functionality and MAF. Hence, it can be argued that at least some of the variants impacting on cancer risk including glioma will be rare. Although the association between the rare variant BRCA2:c.9976A > T, p.(Lys3326Ter) and glioma did not attain statistical significance such an assertion is supported by the established relationship between CHEK2:c.1100delC, p.(Thr367Metfs) and MUTYH:c.536A>G, p.(Tyr179Cys) and MUTYH:c.1187G > A, p.(Gly396Asp) variants which influence the risk of breast and CRC respectively. ${ }^{12,13}$

To our knowledge we have conducted the largest study of the relationship between recurrent PAVs and glioma risk to date. Population stratification is a source of bias in association studies, and although adjustment of test statistics for principal components generated on common SNPs can be applied to genome scans, confounding of rare variants in spatially structured populations is not necessarily corrected by such methods. ${ }^{40}$ Hence a major strength of our study is that it is based on three independent case-control series, thereby minimising biases as a consequence of spatial differences within one data set impacting on conclusions.

No single-variant associations with glioma attained statistical significance after correction for multiple testing. However, we did observe a significant association between variant effect size and predicted functional effect. In this study we have been limited to detecting alleles conferring ORs of 1.6 provided MAF $>0.05(80 \%$ power stipulating $P<10^{-7}$ ) or those with frequencies of $\sim 0.01$ conferring ORs $>2.5$. Hence it is possible that PAVs do have an appreciable contribution to glioma risk but at lower individual effect sizes than previously anticipated, therefore requiring much larger case-control sample sets than we have used herein to identify them.

Testing for a burden of PAVs across genes revealed a significant association between HARS2 and GBM. HARS2 encodes a mitochondrial histidyl tRNA synthetase, mutation of which causes ovarian dysgenesis and sensorineural hearing loss. ${ }^{41}$ Although not 
Table 5 Genes possessing significant burden of germline variants as well as being significantly mutated in GBM and non-GBM tumours

Germline (exome-array)

\begin{tabular}{|c|c|c|c|c|c|c|}
\hline Gene & Variants & $P_{\text {Burden }}$ & Rank $_{\text {Burden }}$ & $P_{S K A T}$ & Rank $_{S K A T}$ & $\begin{array}{c}\text { Tumour } \\
\text { Mutsig Q }\end{array}$ \\
\hline \multicolumn{7}{|l|}{$G B M$} \\
\hline CRAMP1L & 7 & $4.84 \times 10^{-5}$ & 3 & $5.79 \times 10^{-5}$ & 9 & $>0.1$ \\
\hline RBM47 & 3 & $2.29 \times 10^{-4}$ & 8 & $1.57 \times 10^{-5}$ & 6 & $>0.1$ \\
\hline SLC26A6 & 8 & $7.84 \times 10^{-5}$ & 6 & $6.71 \times 10^{-5}$ & 10 & $>0.1$ \\
\hline$A B C B 1$ & 14 & 0.0180 & 282 & 0.235 & 3098 & 0.0154 \\
\hline SEMA3E & 3 & 0.0185 & 290 & 0.0772 & 1054 & 0.0209 \\
\hline DYNC1I1 & 5 & $9.57 \times 10^{-4}$ & 24 & 0.00837 & 181 & 0.071 \\
\hline \multicolumn{7}{|l|}{ Non-GBM } \\
\hline$C P M$ & 5 & $1.05 \times 10^{-4}$ & 18 & $3.58 \times 10^{-4}$ & 11 & $>0.1$ \\
\hline
\end{tabular}

Shown are genes with $P<0.05$ in at least one germline burden test and which either (1) are ranked in the top 20 most associated genes in both tests (highlighted in bold) or (2) have a Mutsig $Q$ score $<0.1$ for the relevant tumour subtype (indicating significantly mutated genes, highlighted in bold).

attaining an exome-wide significant burden of germline variants, additionally of note is $\mathrm{CDH} 18$ and GBM risk. $\mathrm{CDH} 18$ is also significantly mutated in GBM tumours and encodes a cadherin protein involved in cell-cell adhesion. The gene is expressed specifically in the nervous system and has been proposed to regulate neural morphogenesis. ${ }^{42}$

By restricting our analysis to genes implicated in glioma by virtue of somatic mutation or variants recognised to increase risk of other cancers, we constrained the multiple testing problem and upweighted the prior probability for association with glioma. From these analyses we have provided evidence to implicate BRCA2 p.(Lys3326Ter) as well as IDH2 p.(Arg261His) as determinants of glioma risk. IDH2 encodes for the mitochondrial $\mathrm{NAD}(+)$-dependent isocitrate dehydrogenase which is involved in the citric acid cycle. ${ }^{43}$ While IDH2 p.(Arg261His) is not mutated in glioma, $I D H 1$ or $I D H 2$ are commonly mutated in glioma tumours and always involve the arginine residue. ${ }^{44} \mathrm{IDH} 2$ is in chromosome 15q26.1, the location of a previously reported glioma linkage peak. ${ }^{45}$ Modelling of the IDH2 p.(Arg261His) change is shown in Supplementary Figure 4. This amino acid change is predicted to disrupt several salt bridge interactions, which may affect protein activity.

In our study, none of the PAVs genotyped in any of the previously identified glioma GWAS regions showed evidence of association with glioma $\left(n=240 ; P>1.37 \times 10^{-3}\right)$. While accepting that we are constrained by the content of PAVs on the array, this argues against a rare coding variant that is tagged by a SNP contributing significantly to any of the GWAS signals identified.

While aiming to provide a comprehensive survey of recurrent PAVs it is apparent from our analysis that there are a number of issues that will impact on the utility of the Illumina Exome Array. Firstly, a high proportion of the featured SNPs are either monomorphic in Europeans or have a MAF $<0.005$. Secondly, as illustrated by comparison with data from the UK10K sequencing project, $22 \%$ of missense variants with allele counts $>5$ are not featured on the array (11 894 of
54463 variants; Supplementary Table 6). Additionally, only 36\% of PAVs on the array are predicted to be functionally deleterious. Finally, indels are not well represented on the array. Collectively, these observations cast doubt on the ability of the array to provide a comprehensive assessment of the contribution of PAVs to disease risk, highlighting the value of sequence-based approaches to discover new disease variants.

In conclusion, there is increasing evidence that cancer susceptibility is in part mediated through low-frequency variants affecting the amino acid sequence of expressed proteins. Hence genome scans of PAVs represent a complementary strategy to identify disease-causing variants compared to scans based on tagSNPs. Strategies to lessen the multiple testing burden by restricting analysis to PAVs with higher priors affords an opportunity to maximise study power.

\section{CONFLICT OF INTEREST}

The authors declare no conflict of interest.

\section{ACKNOWLEDGEMENTS}

We are grateful to all the patients and individuals for their participation and we also thank the clinicians and other hospital staff, cancer registries and study staff in respective centres who contributed to the blood sample and data collection. For the UK study, we acknowledge the participation of the clinicians and other hospital staff, cancer registries, study staff and funders who contributed to the blood sample and data collection for this study as listed in Hepworth et al (BMJ 2006, 332, 883). For the German study, we are indebted to B Harzheim (Bonn), $S$ Ott and Dr A Müller-Erkwoh (Bonn) for help with the acquisition of clinical data and R Mahlberg (Bonn) who provided technical support. The UK study made use of control genotyping data generated by the Wellcome Trust CaseControl Consortium. A full list of the investigators who contributed to the generation of the data is available from The 1958 Birth Cohort exome chip data was QCd by Kathy Stirrups. Data sharing was organised by the UK Exome-chip consortium. French controls were taken from the SU.VI.MAX study. The German GWA study made use of genotyping data from the Heinz-Nixdorf RECALL study. The HNR cohort was established with the support of the 
Heinz-Nixdorf Foundation. Franziska Degenhardt received support from the BONFOR Programme of the University of Bonn, Germany. We are grateful to all investigators who contributed to the generation of this data set. UK10K data generation and access was organised by the UK10K consortium and funded by the Wellcome Trust. The results here are in part based upon data generated by the TCGA Research Network: http://cancergenome.nih.gov/. In the UK study, funding was provided by Cancer Research UK (C1298/A8362 supported by the Bobby Moore Fund), the Wellcome Trust and the DJ Fielding Medical Research Trust. BK is supported by a PhD studentship funded by the Sir John Fisher Foundation. The UK INTERPHONE study was supported by the European Union Fifth Framework Program 'Quality of life and Management of Living Resources' (QLK4-CT-1999-01563) and the International Union against Cancer (UICC). The UICC received funds from the Mobile Manufacturers' Forum and GSM Association. Provision of funds via the UICC was governed by agreements that guaranteed INTERPHONE's scientific independence (http:// www.iarc.fr/ENG/Units/RCAd.html) and the views expressed in the paper are not necessarily those of the funders. The UK centres were also supported by the Mobile Telecommunications and Health Research (MTHR) Programme and the Northern UK Centre was supported by the Health and Safety Executive, Department of Health and Safety Executive and the UK Network Operators. In France, funding was provided by the Délégation à la Recherche Clinique (MUL03012), the Association pour la Recherche sur les Tumeurs Cérébrales (ARTC), the Institut National du Cancer (INCa; PL046) and the French Ministry of Higher Education and Research. This study was additionally supported by a grant from Génome Québec, le Ministère de l'Enseignement supérieur, de la Recherche, de la Science et de la Technologie (MESRST) Québec and McGill University. In Germany, funding was provided to MS and JS by the Deutsche Forschungsgemeinschaft (Si552, Schr285), the Deutsche Krebshilfe (70-2385-Wi2, 70-3163-Wi3, 10-6262) and BONFOR. Generation of the German control data was partially supported by a grant of the German Federal Ministry of Education and Research (BMBF) through the Integrated Network IntegraMent (Integrated Understanding of Causes and Mechanisms in Mental Disorders), under the auspices of the e:Med research and funding concept (01ZX1314A). MMN received support from the Alfried Krupp von Bohlen und Halbach-Stiftung and is a member of the DFG-funded Excellence Cluster ImmunoSensation.

\section{WEBSITES}

R Core Team (2013) R: A language and environment for statistical computing. R Foundation for Statistical Computing, Vienna, Austria: URL http://www.R-project.org/; Illumina: http://www.illumina.com; Exome chip design: http://genome.sph.umich.edu/wiki/Exome_Chip_ Design; Plink: http://pngu.mgh.harvard.edu/ purcell/plink/; Plink seq: https://atgu.mgh.harvard.edu/plinkseq/; cBioPortal for Cancer Genomics: http://www.cbioportal.org; The Cancer Genome Atlas project: http://cancergenome.nih.gov; UK10K: http://www.uk10k.org/; Wellcome Trust Case Control Consortium (WTCC2): http://www.wtccc. org.uk/; GERP: http://snp.gs.washington.edu/SeattleSeqAnnotation134/; Phast_cons: http://genome.ucsc.edu/cgi-bin/hgGateway); Project HOPE server: http://www.cmbi.ru.nl/hope.

1 Crocetti E, Trama A, Stiller $\mathrm{C}$ et al: Epidemiology of glial and non-glial brain tumours in Europe. Eur J Cancer 2012; 48: 1532-1542.

2 Bondy ML, Scheurer ME, Malmer B et al: Brain tumor epidemiology: consensus from the Brain Tumor Epidemiology Consortium. Cancer 2008; 113: 1953-1968.

3 Louis DN, Ohgaki H, Wiestler OD et al: The 2007 WHO classification of tumours of the central nervous system. Acta Neuropathol 2007; 114: 97-109.

4 Hodgson SV, Foulkes WD, Eng C, Maher ER: A Practical Guide to Human Cancer Genetics, Fourth Edition. Springer: New York, NY, USA, 2014

5 Hemminki K, Tretli S, Sundquist J, Johannesen TB, Granstrom C: Familial risks in nervous-system tumours: a histology-specific analysis from Sweden and Norway. The Lancet Oncology 2009; 10: 481-488.

6 Sanson M, Hosking FJ, Shete S et al: Chromosome 7p11.2 (EGFR) variation influences glioma risk. Hum Mol Genet 2011; 20: 2897-2904.

7 Shete S, Hosking FJ, Robertson LB et al: Genome-wide association study identifies five susceptibility loci for glioma. Nat Genet 2009; 41: 899-904.
8 Wrensch M, Jenkins RB, Chang JS et al: Variants in the CDKN2B and RTEL1 regions are associated with high-grade glioma susceptibility. Nat Genet 2009; 41: 905-908.

9 Botstein D, Risch $N$ : Discovering genotypes underlying human phenotypes: past successes for mendelian disease, future approaches for complex disease. Nat Genet 2003; 33: 228-237.

10 Wang Y, McKay JD, Rafnar T et al: Rare variants of large effect in BRCA2 and CHEK2 affect risk of lung cancer. Nat Genet 2014; 46: 736-741.

11 Woodage T, King SM, Wacholder S et al: The APC I1307K allele and cancer risk in a community-based study of Ashkenazi Jews. Nat Genet 1998; 20: 62-65.

12 Meijers-Heijboer $\mathrm{H}$, van den Ouweland A, Klijn J et al: Low-penetrance susceptibility to breast cancer due to $\mathrm{CHEK} 2\left(^{*}\right) 1100$ delC in noncarriers of BRCA1 or BRCA2 mutations. Nat Genet 2002; 31: 55-59.

13 Al-Tassan N, Chmiel NH, Maynard J et al: Inherited variants of MYH associated with somatic G:C-> T:A mutations in colorectal tumors. Nat Genet 2002; 30: 227-232.

14 Cardis E, Richardson L, Deltour I et al: The INTERPHONE study: design, epidemiological methods, and description of the study population. Eur J Epidemiol 2007; 22: 647-664.

15 Power C, Elliott J: Cohort profile: 1958 British birth cohort (National Child Development Study). Int J Epidemiol 2006; 35: 34-41.

16 Hercberg S, Galan P, Preziosi P et al: The SU.VI.MAX Study: a randomized, placebocontrolled trial of the health effects of antioxidant vitamins and minerals. Arch Intern Med 2004; 164: 2335-2342.

17 Erbel R, Eisele L, Moebus S et al: Die Heinz Nixdorf Recall Studie. Bundesgesundheitsb/ 2012; 55: 809-815.

18 Chubb D, Broderick P, Frampton $M$ et al: Genetic diagnosis of high-penetrance susceptibility for colorectal cancer (CRC) is achievable for a high proportion of familial CRC by exome sequencing. J Clin Oncol 2015; 33: 426-432.

19 McKenna A, Hanna M, Banks E et al: The Genome Analysis Toolkit: a MapReduce framework for analyzing next-generation DNA sequencing data. Genome Res 2010; 20: 1297-1303.

20 DePristo MA, Banks E, Poplin R et al: A framework for variation discovery and genotyping using next-generation DNA sequencing data. Nat Genet 2011; 43: 491.

21 Van der Auwera GA, Carneiro MO, Hartl C et al: From FastQ data to high confidence variant calls: the Genome Analysis Toolkit best practices pipeline. Curr Protoc Bioinform 2013; 11: 1110 11-11 1033

22 Purcell S, Neale B, Todd-Brown K et al: PLINK: a tool set for whole-genome association and population-based linkage analyses. Am J Hum Genet 2007; 81: 559-575.

23 Patterson N, Price AL, Reich D: Population structure and eigenanalysis. PLoS Genet 2006; 2: e190.

24 Price AL, Patterson NJ, Plenge RM, Weinblatt ME, Shadick NA, Reich D: Principal components analysis corrects for stratification in genome-wide association studies. Nat Genet 2006; 38: 904-909.

25 Raj A, Stephens M, Pritchard JK: fastSTRUCTURE: Variational Inference of Population Structure in Large SNP Data Sets. Genetics 2014; 197: 573-589.

26 Wu MC, Lee S, Cai T, Li Y, Boehnke M, Lin X: Rare-variant association testing for sequencing data with the sequence kernel association test. Am J Hum Genet 2011; 89: 82-93.

27 Subramanian A, Tamayo P, Mootha VK et al: Gene set enrichment analysis: a knowledge-based approach for interpreting genome-wide expression profiles. Proc Nat Acad Sci USA 2005; 102: 15545-15550.

28 Lawrence MS, Stojanov P, Polak P et al: Mutational heterogeneity in cancer and the search for new cancer-associated genes. Nature 2013; 499: 214-218.

29 Gao J, Aksoy BA, Dogrusoz U et al: Integrative Analysis of Complex Cancer Genomics and Clinical Profiles Using the cBioPortal. Sci Signal 2013; 6: pl1.

30 McLaren W, Pritchard B, Rios D, Chen Y, Flicek P, Cunningham F: Deriving the consequences of genomic variants with the Ensembl API and SNP Effect Predictor. Bioinformatics 2010; 26: 2069-2070.

31 Ng PC, Henikoff S: Predicting deleterious amino acid substitutions. Genome Res 2001; 11: 863-874.

32 Adzhubei I, Jordan DM, Sunyaev SR: Predicting functional effect of human missense mutations using PolyPhen-2. Curr Protoc Hum Genet 2013; Unit 7: 20.

33 Gonzalez-Perez A, Lopez-Bigas N: Improving the assessment of the outcome of nonsynonymous SNVs with a consensus deleteriousness score, Condel. Am J Hum Genet 2011; 88: 440-449.

34 Venselaar H, Te Beek TA, Kuipers RK, Hekkelman ML, Vriend G: Protein structure analysis of mutations causing inheritable diseases. An e-Science approach with life scientist friendly interfaces. BMC Bioinform 2010; 11: 548.

35 Cooper GM, Stone EA, Asimenos G et al: Distribution and intensity of constraint in mammalian genomic sequence. Genome Res 2005; 15: 901-913.

36 Siepel A, Bejerano G, Pedersen JS et al: Evolutionarily conserved elements in vertebrate, insect, worm, and yeast genomes. Genome Res 2005; 15: 1034-1050.

37 Michailidou K, Hall P, Gonzalez-Neira A et al: Large-scale genotyping identifies 41 new oci associated with breast cancer risk. Nat Genet 2013; 45: 353-361, 361e351-352.

38 Han FF, Guo CL, Liu LH: The effect of CHEK2 variant I157T on cancer susceptibility: evidence from a meta-analysis. DNA Cell Biol 2013; 32: 329-335.

39 Cantor SB, Bell DW, Ganesan S et al: BACH1, a novel helicase-like protein, interacts directly with BRCA1 and contributes to its DNA repair function. Cell 2001; 105: 149-160.

40 Mathieson I, McVean G: Differential confounding of rare and common variants in spatially structured populations. Nat Genet 2012; 44: 243-246.

41 Pierce SB, Chisholm KM, Lynch ED et al: Mutations in mitochondrial histidyl tRNA synthetase HARS2 cause ovarian dysgenesis and sensorineural hearing loss of Perrault syndrome. Proc Natl Acad Sci USA 2011; 108: 6543-6548. 
42 Shibata T, Shimoyama Y, Gotoh M, Hirohashi S: Identification of human cadherin-14, a novel neurally specific type II cadherin, by protein interaction cloning. J Biol Chem 1997; 272: 5236-5240.

43 Oh IU, Inazawa J, Kim YO, Song BJ, Huh TL: Assignment of the human mitochondrial NADP(+)-specific isocitrate dehydrogenase (IDH2) gene to $15 \mathrm{q} 26.1$ by in situ hybridization. Genomics 1996; 38: 104-106.

44 Yan $\mathrm{H}$, Parsons DW, Jin G et al: IDH1 and IDH2 mutations in gliomas. N Engl J Med 2009; 360: 765-773.

45 Paunu N, Lahermo P, Onkamo P et al: A novel low-penetrance locus for familial glioma at 15q23-q26.3. Cancer Res 2002; 62: 3798-3802.
This work is licensed under a Creative Commons Attribution 4.0 International License. The images or other third party material in this article are included in the article's Creative Commons license, unless indicated otherwise in the credit line; if the material is not included under the Creative Commons license, users will need to obtain permission from the license holder to reproduce the material. To view a copy of this license, visit http:// creativecommons.org/licenses/by/4.0/

Supplementary Information accompanies this paper on European Journal of Human Genetics website (http://www.nature.com/ejhg) 